\title{
KOMMUNIKATIVE FUNKTIONEN \\ DER EINFACHEN AUSSAGESÄTZE IN DER GESTALTSPRACHE DER DEUTSCHEN SPIELFILME
}

\begin{abstract}
Анотація. У статті представлено результати дослідження комунікативних функцій простих розповідних речень, реалізованих у персонажному діалогічному мовленні німецьких художніх фільмів. Речення розглядається як одиниця мовного та мовленнєвого рівнів, адже має чітко визначену структурну будову, яка фіксує його зміст, при цьому здатна реалізувати комунікативні наміри мовців.

Просте розповідне речення в німецькій мові $є$ двоскладною мовною структурою, дієслово-присудок у якій займає другу позицію. Проте, реалізуючись у діалозі, таке речення підкоряється не тільки мовним законам, але й комунікативним намірам співрозмовників. Вектор дослідницької уваги в представленій науковій праці спрямований на двосторонній підхід до розгляду простого розповідного речення, яке, з одного боку, слугує засобом реалізації режисерського задуму, а з іншого, $є$ інструментом комунікативної взаємодії акторів, що намагаються уподібнити своє мовлення до природного.

Під час вивчення враховуються також позамовні фактори (умови позамовної дійсності, що впливають на зміст, структуру, а також функції простого розповідного речення): комунікативні наміри мовців та міжособистісні стосунки, які склались між ними. Вивчення комунікативних функцій простого розповідного речення у статті здійснено 3 урахуванням таких позамовних факторів, як конфліктність та формальність. Конфліктність та формальність розрізняються характером стосунків співрозмовників та емоційністю їх мовлення. Конфліктність передбачає конфронтативні відносини, засновані на емоційній неприязні мовців один до одного, метою яких є протиставлення позиції одного учасника дискусії, поглядам іншого і навпаки. Експресивність мовлення комунікантів при цьому впливає на вибір мовних засобів, вираження яких повністю підкоряються меті мовця. У зв'язку з цим структуру речення та його зміст можуть бути змінені. Формальний характер стосунків, навпаки, максимально позбавлений емоцій та спрямований на конструктив, а речення в ситуації формальної взаємодії слугують засобом донесення інформації та максимально позбавлені впливу емоцій на їхнє вираження.
\end{abstract}

Ключові слова: речення, просте розповідне речення, персонажне мовлення, комунікативна функція.

Problemstellung. Die moderne Linguistik konzentriert sich immer wieder auf die Untersuchung von Spracheinheiten, die als Mittel der menschlichen Kommunikation in künstlerischen Bedingungen (Filmen, Ausstellungen usw.) $[1 ; 3 ; 8 ; 9]$ ausgeübt ist.

Die GS (Gestaltsprache) ist ein Bestandteil des Films, ein Hauptmittel der Realisation der Idee von den Autoren. Die Autoren des sprachlichen Kodes im Film sind die Autoren, Regisseure und Schauspieler. Für in dem Film realisierter GS sind einige typi- sche Merkmale charakteristisch: 1) die Ähnlichkeit der in dem Film dargestellten Kommunikation der menschlichen Rede in der Wirklichkeit; 2) zwei Autoren des Textes der GS (der Drehbuchautor und Regisseur, manchmal auch Schauspieler); 3) zwei Adressaten (im Film - der andere Gestalt, außer des Films - der Zuschauer). Diese Besonderheiten des Films können auch die syntaktischen Besonderheiten der sprachlichen Einhdeiten, die als Mittel der Kommunikation gebraucht wird, beeinflussen.

Analyse der aktuellen Forschungsergebnisse und Veröffentlichungen. Mit dem Problem der kommunikativen Rolle der Sprachelementen waren viele ukrainische (F.S. Bazevitsch, L.R. Bezugla, Y.O. Dzekun) als auch russische (A.R. Atchipov, N.D. Arutiunova, Y.A.Vodnev, V.D. Devkin, L.M. Michailov) und deutsche (H. Welman, G. Zifonnun) Forscher beschäftigt.

Vorstellung des Hauptmaterials. Die sprachliche Bezeichnung des Minimums der kommunikativen Information in den gespielten Situationen, wie in der natürlichen Sprache, ist der Satz. Der Satz ist Instrument der Widerspiegelung des menschlichen Gedankens [5, s. 45]. Die Realisationsform des Satzes unterordnet sich den Gesetzen der Sprache. In der Interpretation des Begriffs „Satz" schließen wir uns der Meinung von Pr. Potscheptsov G.G. an. Der Wissenschaftler bezeichnet den im Dialog realisierten Satz als minimale syntaktische Struktur, die in Akten der Sprachkommunikation verwendet wird, und realisiert ein bestimmtes Strukturschema [4, s. 165]. Das bedeutet, dass in der Rede realisierte Sätze unter dem Einfluss von außersprachlichen Faktoren eine Möglichkeit X-beliebige kommunikative Funktionen ausüben haben.

Eine der wichtigsten sprachlichen Instrumente der menschlichen Kommunikation sind die einfachen Aussagesätze. Einfacher Aussagesatz ist eine Einheit der Sprache, die inhaltlich beendete Information ausdrückt [2, s. 178]. Diese Sätze gehören zur Gruppe der Verbzweitsätze (Sätze, in denen das Verb-Prädikat sich in der zweiten Position befindet) [7, s. 903]. Was die deutsche Sprache betrifft, entscheidende Rolle in der Struktur des Satzes ein Prädikat spielt, der erste oder dritte Position einnehmen kann. Die anderen Satzglieder können ,freie“ Plätze besitzen [6, s. 128]. Diese Charakterzüge bestimmen zwei Strukturarten der einfachen Aussagesätze im Deutschen, die wir mit der Hilfe von solchen Modellen darstellen können: 1) $\mathrm{S}+\mathrm{P}+(0)$ - Sophie liest (ein Buch); 2) $\mathrm{Adv}+\mathrm{P}+\mathrm{S}+(0)$ Zu Hause liest Sophie ein Buch, wo S (Subjekt), P (Prädikat), $\mathrm{O}$ (Objekt), Adv (Adverbialbestimmung) sind.

Aber in der Gestaltsprache (GS) der deutschen Filme gebraucht man auch die Aussagesätze, die den anderen Modellen entsprechen. Die Veränderungen in der Satzstruktur sind von den außersprachlichen Faktoren verursacht, z.B. von dem Wunsch des Menschen den Mitsprecher zu beeinflussen. 
Das Ziel des Artikels besteht darin die kommunikativen Funktionen der einfachen Aussagesätze in der GS der modernen deutschen Filme zu forschern. Die Aufgabe des Artikels ist Bestimmung und Interpretation der Funktionsbesonderheiten der einfachen Aussagesätze in der GS der modernen deutschen Filme.

Das Objekt der Forschung ist eine einfache Aussagesatz der deutschen Sprache; der Gegenstand ist Besonderheit der kommunikativen Funktionen von oben genannten Sätze in der GS der deutschen Filme.

Das Material der Forschung bilden 1230 einfache Aussagesätze, die aus den modernen deutschen Spielfilmen ausgewählt werden.

Die Aktualität des Artikels besteht darin, dass untersuchtes Problem sich in den Rhamen der modernen linguistischen Studien befindet und ist dem Erlernen der Funktionen der Spracheinheiten in dem Kommunikationsprozess gewidmet.

Die wissenschaftliche Neuheit der Arbeit ist die Bestimmung der Funktionsbesonderheiten der einfachen Aussagesätze in dem Kommunikationsprozess.

In unserer Forschung bestimmen wir alle strukturellen Modelle der in der GS gebrauchten einfachen Aussagesätze und entscheiden danach, ob die Veränderung der Struktur die kommunikativen Funktionen dieser Sätze beeinflusst. Damit beachtet man auch die Verhältnisse zwischen den Gesprächspartnern, die sich von folgenden Kriterien abhängen: Formalität (nähere oder offizielle Verhältnisse) und Konflikt (freundliche oder feindliche Verhältnisse).

In der GS der deutschen Filme die einfachen Aussagesätze verwendet man für den Bericht neuer Information, als auch für die Versorgung der kommunikativen Tätigkeit der Gesprächspartner. In dieser Hinsicht können die Aussagesätze in zwei Gruppen unterteilt werden: a) Informationsmittel; b) Mittel der kommunikativen Wirkung. Im ersten Fall übt der Aussagesatz seine direkten Funktionen aus. In der zweiten kann er Anzeichen von anderen Satzarten (interrogativen oder imperativen) bekommen, d.h. indirekte Funktionen (Anforderung, Ersuchen, Aufruf, Warnmeldung u. a.) ausüben.

Wie die Beispiele zeigen, sind einfache Aussagesätze in der GS der deutschen Filmen Hauptmittel Bericht, Erklärung, Überzeugung und Nachweis auszudrücken. Oben genannte Funktionen können sich aufgrund subjektiven und objektiven Bedingungen verändern: 1) die Einstellung des Sprechers zur Information und zum Begleiter; 2) sein emotionaler Zustand; 3) die Wichtigkeit der Nachrichtenbedeutung das Gespräch fortzusetzen; 4) Abhängigkeit des Satzes vom Kontext der früheren Aussagen.

Die Untersuchung zeigte, dass der Ausdrucksmittel der unbekannten Information im GS einfache Aussagesätze, die dem Modell $\mathrm{S}+\mathrm{P}+(\mathrm{O})$ : Peter liest (ein Buch) entsprechen, sind. Solche kommunikative Funktionen können sie in den verschiedenen Situationen, insbesondere in den nicht formellen Konfliktsituationen, wo Interaktion von keinen Regeln (sozialen, sprachlichen) eingestellt ist, erwerben.

In dem Film „Die Welle“ stellte man den Dialog von dem Mann und seiner Frau vor. Beide sind Lehrer in einer Schule aber haben unterschiedliche Ansichten an die Erziehung der Jugendlichen. Die Frau versteht nicht den Optimismus des Mannes, der durch die positiven Veränderungen in dem Verhalten der Schüller unter dem Einfluss der Gemeinschaft „die Welle“ hervorrufen ist. Sowieso versucht Rainer die Frau zu überzeugen ihm zu glauben.

Rainer: Kaschi ist total motiviert. Die Kids machen gerade ne richtige Veränderung durch.
Frau: Klar. Sieht man ja auch. Ihr mit euren weißen Hemden.

Rainer: Ja okay, ich hab vergessen mich umzuziehen.

Frau: Im Kollegium reden sie auch schon über dich.

Rainer: Das ist mir egal. Das haben die immer gemacht. (Die Welle, 00:46).

Rainer versucht positive Seite seiner neuen Methodik in der Jugende „rziehung vorzustellen. Mit den Sätzen „Kaschi ist total motiviert" und "Die Kids machen gerade ne richtige Veränderung durch" berichtet er der Frau über die Veränderungen, die mit den Jungen passierten.

In dem selben Dialog verwendet man die Sätze mit eingeschränkter syntaktischen Struktur, die auf Grund der Modellen S+NP, wo NP nominales Prädikat ist: "Ihr mit euren weißen Hemden" und $\mathrm{P}+\mathrm{S}+(\mathrm{Si})$ : "Sieht man ja auch" verbalisier sind. Diese Sätze dienen als Ausdrucksmittel der Wunsch der Frau zu berichten, dass der Mann in seiner Vermutungen neue hocherzogene Generation zu entdecken irrt. Anke verändert die Struktur des Satzes „Sieht man ja auch“, um den Mann zu beeinflussen. Es wandelt ihr Satz auf expressive Aussage um. Der Satz "Ihr mit euren weißen Hemden" ist ein Mittel der Verbalisierung des emotionellen Einflusses auf Rainer, womit will die Frau ihn überzeugen, die Verwendung von weißen Hemden abzulehnen. Die Frau ist überzeugend, dass diese Attribute "Welle" in der Sekte verwandeln und dass Rainer sein Experiment beenden soll.

Als sprachlicher Mittel der Realisierung des Beweisen in GS können die Sätze, die der Modelle $\mathrm{NP}(\mathrm{O})+\mathrm{P}+\mathrm{S}$ entsprechen, dienen: Gut liest Peter. Solche kommunikative Funktion übt man die Sätze in formalen Konfliktsituationen aus. Der in die Präposition gestellter nominaler Prädikat dient dafür, die Aufmerksamkeit des Sprechers auf dem bestimmten Fakt zu akzentuieren. Dank solcher Unternehmen, bekommt der Sprecher eine Möglichkeit aufgrund der Vernichtung der Gedanken des Mitsprechers seine Rechtlichkeit zu beweisen.

In den nicht formaler Konfliktsituation, die ein Gespräch von zwei Brüdern darstellt, gebraucht man der Satz, der aufgrund von oben genannter Modelle realisiert wird, mit dem Ziel der Schuld von Sebastian in dem Tode der Mutter, die im Moment des Geburten gestorben ist, zu beweisen.

Bruder: Die Hasen ja. Und wen hast du noch umgebracht?

Sebastian: Ich weiß nicht.

Bruder: Du weißt nicht?

Sebastian: Nein.

Bruder: Mama hast du umgebracht.

Sebastian: Was?

Bruder: Dafür entschuldigst du dich! (Wer früher stirbt, ist länger tot, 00:10).

Das Herz des Bruders tut ihm bis jetzt weh wegen des Mutterstode, Sebastian aber erinnert ihm darüber immer wieder. Dieses Mal hat er drei beste Kaninchen umgebracht. Mit der Hilfe von veränderter Wortfolge versucht der Bruder zu beweisen, dass Sebastian in allen von diesen Toden schuldig ist. Die Aufmerksamkeit von dem Gesprächspartner auf die Tatsachen, die in der Präposition verwendet sind: "Mama hast du umgebracht"; "Dafür entschuldigst du dich", orientiert ist.

Analyse von der GS des deutschen Films zeigten, dass die implizite Ausdruck des Ersuchens kann mit der Hilfe der Sätze gemacht werden, die auf der Basis von dem strukturellen Modell $\mathrm{S}+\mathrm{P}+(\mathrm{O})$ vorgestellt werden. Aber für Realisation solcher Funktion müssen einige Voraussetzungen gehalten werden: das Subjekt ist in 
1. Person Sg. oder Pl. dargestellt und Prädikat ist von dem Verbindung der Vers brauchen und dem Vinf (Infinitiv), vorgestellt. Kommunikative Funktion des Ersuchens können solche Sätze in den nicht formalen konfliktlosen Kommunikationsbedingungen zu erwerben.

Kommunikative Funktion des Ersuchens erwarbt der Satz "Ich brauch Hunderttausend Mark" (Lola rennt, 00:22), der von Lolla im Gespräch mit ihrem Vater gesprochen wurde. Die Verwandten (Vater und Tochter) besprechen eine Möglichkeit der finanziellen Unterstützung für das Mädchen. Lolla versucht das Geld, das das Leben ihres Freundes retten können, zu bekommen. Sie erzählt dem Vater, dass sie seine finanzielle Unterstützung braucht, "Ich brauch Hunderttausend Mark" (Lola rennt, 00:22). Obwohl sie fragt nach Geld, die Information aber klingelt wie Bitte um Hilfe. Hinsichtlich ähnlicher Situationen in der GS der deutschen Spielfilme kann man sagen, dass oben beschrieben einfache Aussagegesätze eine Funktion des Ersuchens bekommen können.

Im demselben Dialog mit der Ziel der Anforderung das Geld zu bekommen, verwendet Lola einfacher Aussagesatz, der auf Grund des Modells $\mathrm{S}+\mathrm{MV}+(\mathrm{E})+\mathrm{Vinf}:$ „Du musst mir helfen“ (Lola rennt, 00:24), wo MV (Modalverb) ist, gebildet ist. Imperative Eigenschaften können diese Sätze dank der grammatischen Form der Hauptsatzglieder bekommen. Dazu muss das Subjekt durch das Personalpronomen der 2. Pers. Sg. oder 3 Pers. Pl und Prädikat durch eine Kombination aus dem Modalverb im Sinne „Notwendigkeit" (sollen / müssen) und dem Hauptverb im Infinitiv ausgedrückt sein. Z.B. bei dem Gebrauch des Satzes ,Musst Du mir helfen“"versucht Lola die Vatersgefühle zu erwachen. Solcher Weise fordert das Mädchen seine Bitte auszuüben.

Model $\mathrm{S}+\mathrm{P}+(\mathrm{O})$ kann in der GS verwendet werden um den Anruf zu äußern. Solche kommunikative Funktion können die Sätze im Falle, wenn die Hauptsatzglieder durch bestimmte Satzkomponente dargestellt sind: du/wir/ihr/Sie $+\mathrm{P}+(\mathrm{O})$. Kommunikative Funktionen des Aufrufs verlangt auf Grund von diesem Model gebildeter Satz im Rahmen der formalen konfliktlosen Gesprächssituationen. Z.B. in dem Film „Im Juli“ eines der Mädchen versucht Mitschülern davon zu überzeugen, das Klassenzimmer organisierend zu verlassen. Sie motiviert ihre Entscheidung mit der Meinung, dass vor den Ferien die Kinder nicht so lange lernen dürfen.

Kira: Herr Bannier, wir können doch nicht in der letzten Stunde vor den Ferien Unterricht machen.

Daniel: Was sollen wir denn sonst machen?

Kira: Wir machen Schluss. Tschüß, Herr Bannier.

Daniel: Tschü $\beta$, Kira. Frohe Ferien. (Im Juli, 00:17).

Die Aussage des Mädchens ist auf Konsolidierung der Mitschüler ausgerichtet. Sie klingelt wie ein Ausruf zusammen zu versammeln und den Klassenraum zu verlassen. Radikalität der Entscheidung ist in der maximalen Einschränkung der Satzform ausgedrückt. Der Satz "Wir machen Schluss“ enthält nur die Hauptsatzglieder und hat keine Elemente, die seine Struktur und Inhalt erweitern können.

Imperative Bedeutung können auch einfache Aussagesätze erwerben, die auf Grund des Models $\mathrm{S}+\mathrm{P}+(\mathrm{O})$ gebildet sind. Diese Bedeutung erwerben sie im Fahl, wenn das Subjekt durch unpersönliches Pronomen (Es+ist +0$)$ ausgedrückt wird. In den formalen konfliktlosen Bedingungen werden sie für die Warnmeldung gebraucht aber nur im Fahl, wenn es nötig ist ein Zweckmäßigkeit / Nichtzweckmäßigkeit zu erklären.

Formale konfliktlose kommunikative Situation ist in dem Film „Good bye, Lenin“ präsentiert. Während des Gesprächs mit dem
Arzt, erklärt der Sohn einer Patientin, die lange Zeit im Koma lag, warum er seine Mutter aus dem Krankenhaus nicht abholen muss.

Arzt: Sie müssen jegliche Aufregung, ja nur die Gefahr einer Aufregung von Ihrer Mutter fernhalten. Und wenn ich das sage, dann meine ich jedwede Aufregung, Herr Kerner.

Alex: Jedwede Aufregung.

Arzt: Es ist lebensbedrohlich. (Good bye, Lenin, 00:22).

Der Junge hat ein Wunsch seiner Mutter etwas Angenehmes zu machen, dazu will er sie zu Hause zu kehren. Aber der Arzt ist nicht sicher, dass sein Versprechen für eine kranke Frau zu kümmern der Junge genau ausüben wird, wie er dies sicherstellt. Also der Mann verwarnt den Jungen das Leben der Mutter nicht zu riskieren: „Es ist lebensbedrohlich".

Die einfachen Aussagesätze mit den Modalverben werden in dem kommunikativen Prozess die Funktionen: der Genehmigung, die Wahrnung ausüben. Für die Realisation der Genehmigung können solche Sätze gebraucht werden, die den Modellen $\mathrm{MV}+\mathrm{S}+(\mathrm{E})+$ Vinf oder $\mathrm{O}+\mathrm{Vmod}+\mathrm{S}+(\mathrm{Si} / \mathrm{O})+($ Vinf $)$ entsprechen, wo MV - Modalverb, E - Ergänzung, Si - Kommunikative Situation. Dieser Funktion erwerben die Sätze, prädikatives Zentrum von welchen durch die Verbindung von dem Hauptverb in Modalverb mit der Bedeutung der Möglichkeit (können oder dürfen) gebildet wird.

Die Funktionen der Genehmigung erwerben oben genannte Sätze in den nicht formalen Konfliktsituationen. Zum Beispiel im Film „Mondscheinkinder“, wenn ein Junge auf Bitte des Mädchens den Fahrrad zu reparieren mit der Genehmigung der Verkehrsmittel bei ihm zu lassen antwortet.

Simon: Kannst du dein Fahrrad lassen. Die Gangschaltung ist kaputt. Kostet'n Zehner.

Lisa: (antwortet nichts). (Mondscheinkinder, 01:08).

Expressivität des Ausdrucks von Simon erreicht man dank der Präpositionsstellung des Modalverbs. Der Junge hat kein Wunsch Lisa zu helfen und um sein Ärger zu zeigen, umstrukturiert er den Satz solcher Weise, dass am Anfang sich ein Modalverb befindet. Damit zeigt Simon, dass er erlaubt, das Fahrrad zu lassen.

Die Sätze mit indirekter Wortfolge, die dem Modell $\mathrm{O}+\mathrm{MV}+\mathrm{S}+(\mathrm{Si} / \mathrm{O})+($ Vinf $)$ entsprechen, in den nicht formalen konfliktlosen Situationen könne auch die Warnung zum Ausdruck bringen. Solche kommunikative Funktionen erwirbt ein Satz im Fahl, wenn Modalverbs „Notwendigkeit“ ausdrückt, d.h. durch Verben sollen / müssen repräsentiert ist. Warnung erwerben Sätze, in denen der Sprecher über die Handlungen der anderen entrüstet und Absicht die Möglichkeit von unerwünschten Situationen in der Zukunft zu verhindern akzentuiert.

Mit dem Satz Das muss ich Dr. Mauer sagen (Mondscheinkinder, 01:16), der dem Modell O+MV+S+(Si/O)+Vinf entspricht, äußert Krankenbruder seinen Unzufriedenheit mit dem Benehmen von zwei Jugendlichen, die aus dem Krankenhaus das an Hautkrebs kranke Kind gestohlen haben.

Krankenbruder: Da seid ihr ja. Ihr seid völlig verrückt geworden. Paul braucht unbedingt Ruhe. Das muss ich Dr. Mauer sagen. Jetzt ab ins Bett.

Die Kinder: (gehen einfach) (Mondscheinkinder, 01:16).

Der Mann kann seine Gefühle nicht zurückhalten, weil das unverantwortliche Verhalten von Kindern Tod des kleinen Jungens verursachen konnte. Der Krankenbruder warnt die Kinder, dass er über den Vorfall den Doktor berichtet: Das muss ich Dr. Mauer sagen. Sein Versprochen muss für die Kinder wie eine Gefahr nicht 
mehr den kleinen Bruder zu sehen klingeln, weil der Doktor ihnen verboten hat mit dem Paul draußen zu gehen.

Die Ergebnisse von der Untersuchung zeigen, dass in der GS der deutschen Spielfilme gebrauchte einfache Aussagesätze nicht nur als Informationsträger betrachtet sind, sondern auch als Kommunikationsmittel. Die wichtigste Voraussetzung für Veränderung der syntaktischer Struktur und kommunikativer Funktion des einfachen Aussagesatzes bildet die außersprachliche Situation, wo sich die Gesprächspartner befinden. Dank der extralinguistischen Faktoren verwandeln sich einfache Aussagesätze in die Kommunikationsmittel. Die Schauspieler gebrauchen diese sprachlichen Einheiten um den kommunikativen Einfluss auf die Zuschauer zu machen.

Je nach der Kommunikationsbedingungen und den Kommunikationsabsichten der Gesprächspartner, können einfache Aussagesätze in ihren direkten Funktionen, wie Bericht, Erklärung, Überzeugung und Nachweis, gebraucht sein; oder indirekte Funktionen, wie Beweisen, Anfrage, Anforderung, Erlaubnis oder Annahmen ausüben.

\section{Literatur:}

Dissertation:

1. Беляева П.А. Лингвистический анализ диалогической речи в художественном тексте : дисс. на соиск. ученой степени канд. филол. наук : спец. 10.02 .04 «Германские языки». Москва, 2005. 172 с.

\section{Monographien:}

2. Вельман Ханс. Грамматика немецкого языка. Звук. Слово. Предложение. Текст. М. : Московский Лицей, 2009. 568 с.

3. Лагутин В.И. Проблемы анализа художественного диалога (к прагмалингвистической теории драмы). Кишинев : Штиица, 1991.99 c.

4. Почепцов Г.Г. Теоретическая грамматика современного английского языка. М. : Высш. школа, 1981. 283 с.

5. Юдакин А.П. Развитие структуры предложения в связи с развитием структуры мысли. М. : Наука, 1984. 168 с.

6. Engel Ulrich Syntax der deutschen Gegenwartssprache. Berlin : E. Schmidt, 2009. 309 S.

\section{Aufsätze in Sammelbänden:}

7. Duden. Die Grammatik. Mannheim-Leipzig-Wien-Zürich: Dudenverlag, 2006. 1343 S.

\section{Aufsätze in Zeitschriften:}

8. Бехта I.A. Дискурсна зона персонажа у фактурі художнього текста // Наукові записки. Серія «Філологічна» / ред. колегія. Острог : Вид-во нац. ун-ту «Острозька академія». Вип. 29. С. 248-250.

9. Борисенко Н.Д. Персонажне мовлення як відображення соціально-статусних характеристик комунікантів на матеріалі британської драми // Мовні і концептуальні картини світу / відп. ред. O.I. Чередниченко. К. : ВПЦ «Київський університет», 2013. № 43. C. 159-165.

Zitierte Quellen:

1. Good bye, Lenin! (Spielfilm), Regie: Wolfgang Becker. Deutschland: X Filme / WDR/Arte Produktion, 2003, 120 min.

2. Im Juli, Regie (Spielfilm), Regie: Fatih Akin. Deutschland: Bavaria Film International, Wüste Filmproduktion mit Filmförderung Hamburg, 2000. 95 Min.
3. Mondscheinkinder (Spielfilm), Regie : Manuela Stacke, Deutschland : Mathias Film, Luna Film, 2006. 87 min.

4. Lola rennt (Spielfilm), Regie: Tom Tykwer. Deutschland: X-Filme / Creative Pool / WDR / Arte, 1998. $81 \mathrm{~min}$.

5. Wer früher stirbt, ist länger tot (Spielfilm), Regie: Markus Haarfanger, Deutschland : Mathias Film, Luna Film, 2006. 106 min.

6. Die Welle Spielfilm (Spielfilm), Regie: Dennis Gansel. Deutschland : Rat Pack Filmproduktion GmbH, Constantin Film Produktion, 2008. 107 Min.

Orobinska R. Communicative functions of simple narrative sentences in the characters speech of German feature films

Summary. The article summarizes the results of the study of the communicative functions of simple narrative sentences implemented in the characters' dialogical speech in German feature films. The sentence is considered as a unit of speech and a speech level, since it has a clearly defined structure, which captures its content, while being able to realize the communicative intentions of the speakers. A simple narrative sentence in the German language is a complete linguistic structure; the verb-predicate occupies the second position.

However, being realized in a dialogue such a sentence obeys not only linguistic laws, but also the communicative intentions of the interlocutors. The vector of research attention in the presented scientific work is aimed at a twoway approach to the consideration of a simple narrative sentence, which, on the one hand, serves as a means of implementing the director's plan, and on the other hand, is an instrument of communicative interaction of actors trying to assimilate their speech to the natural.

During study, extra-language factors (conditions of extracurricular validity affecting the content, structure, and functions of simple narrative sentences) are taking into account: communicative intentions of the speakers and interpersonal relationships that have developed between them. The study of the communicative functions of a simple narrative sentence in the article is taking into account such extralinguistic factors as conflict and formality. Conflict and formality differ in the nature of the interlocutors' relations and the emotionality of their speech. Conflict involves confrontational relations, based on emotional hostility of speakers to each other, whose purpose is to oppose the position of one participant in the discussion, the views of the other and vice versa.

The expressiveness of speech communicants thus influences the choice of linguistic means, whose expressions are subordinated to the speaker's purpose. In this regard, the structure of the sentence and its contents may be changed. The formal nature of the relationship, on the contrary, is as much as possible emotionally devoid and constructive and sentences in the formal interaction situation serve as a means of communicating information and, as far as possible, deprived of emotional influence on its expression.

Key words: sentence, simple narrative sentences, character's speech, communicative function. 